\title{
Fusión de labios mayores: causa excepcional de obstrucción urinaria
}

\author{
López Aramburu MA, Rosa Arias J, Peña Pérez P. \\ Servicio de Urología. Hospital Santiago Apóstol. Miranda de Ebro. Burgos. \\ Actas Urol Esp. 2008;32(10):1037-1038
}

\section{RESUMEN}

FUSIÓN DE LABIOS MAYORES: CAUSA EXCEPCIONAL DE OBSTRUCCIÓN URINARIA

Presentamos una caso de fusión labial en una mujer anciana. La paciente fue atendida en el servicio de urología al referir síntomas miccionales. El diagnóstico se realizó por la mera inspección de la región vulvar. Bajo anestesia se practicó tratamiento quirúrgico con disección roma, y con la aplicación de pomadas de estrógenos al alta, se consiguió un resultado exitoso. El déficit estrogénico parece estar en la base de este raro proceso al que se pueden añadir circunstancia como liquen esclero atrófico, diabetes, o disminución de la actividad sexual entre otros.

Palabras clave: Fusión labial. Obstrucción urinaria.

\section{ABSTRACT \\ LABIAL ADHESIONS: UNUSUAL CAUSE OF URINARY OBSTRUCTION}

A case of labial adhesions in an elderly woman is reported. The patient was admitted in the urology department since she related urinary symptoms. The diagnosis was been made by simple inspection of the genital region. Under anaesthesia, surgical procedure was done by sharp dissection, and dressing ointment was recommended at discharge. An excellent result was obtain. The estrogens deficiency seems to be the origin of this rare disease and it is not unusual the presence of another circumstances like lichen sclerous et atrophicus, diabetes mellitus or decreasing sexual activity.

Keywords: Labial adhesions. Urinary obstruction.

$\mathrm{L}$ a fusión labial completa o incompleta es una entidad de relativa frecuencia en niñas ${ }^{1,2}$. En la mujer adulta y anciana se convierte en una excepcionalidad y más si tenemos en cuenta que el motivo de la consulta es, en estos raros casos, una sintomatología urológica y no ginecológica ${ }^{3}$.

Presentamos una caso de una mujer anciana que presentó severos síntomas miccionales causados por una fusión de labios mayores. Únicamente un mínimo agujero permitía la eliminación de orina.

\section{CASO CLÍNICO}

Paciente de sexo femenino de 86 años de edad, diabética en tratamiento con antidiabéticos orales que acude a urgencias de nuestro Hospital por presenta síndrome disúrico de larga evolución. En el interrogatorio destaca la presencia de micciones prolongadas, dolorosas, y con importante goteo postmiccional.

En la exploración (Fig. 1) sorprende la existencia de una fusión de labios mayores casi total, identificándose un pequeño orifico a nivel medio, único lugar por donde la paciente elimina la orina al exterior. El estudio urológico por imagen (ecografías) no demostró anomalías de interés. Bajo anestesia raquídea se procede a disección roma de labios mayores lo que se logra con relativa facilidad (Fig. 2). Al alta se indica a la paciente y la necesidad de higiene esmerada de toda la zona genital, con aplicación local de pomada de estrógenos. La paciente recupera micciones fisiológicas confortables desapareciendo toda la sintomatología que motivó su ingreso. 


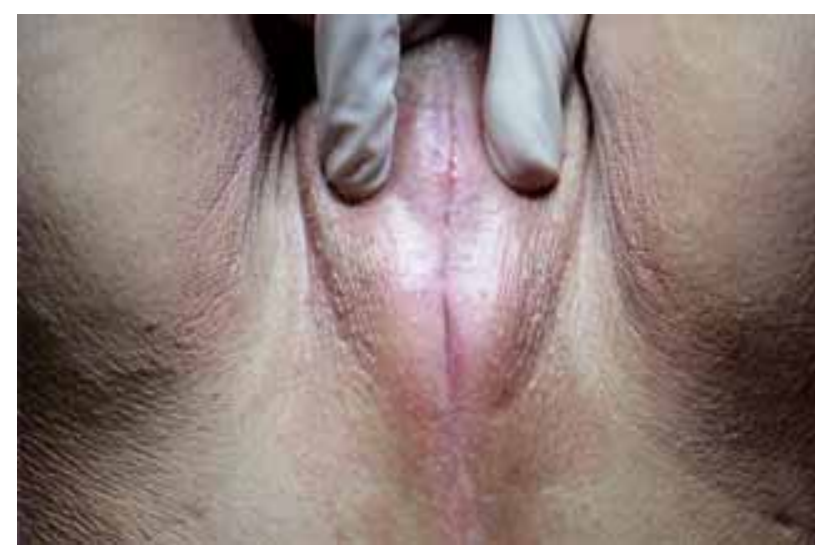

FIGURA 1: La fusión labial es ostensible a la mera exploración.

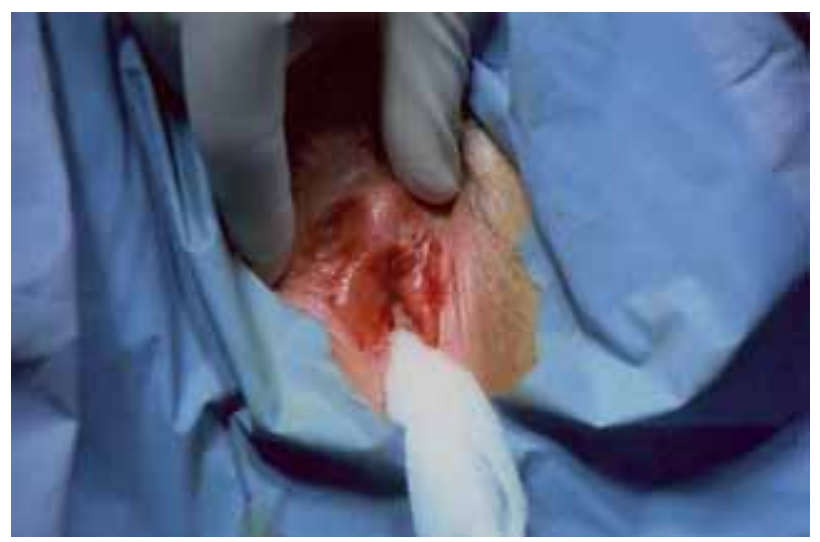

FIGURA 2: Aspecto de la región genital tras separación quirúrgica de los tejidos.

\section{DISCUSION}

La fusión de labios mayores en la mujer adulta es una rara entidad, y en la revisión de la literatura se encuentran normalmente casos aislados, destacando la abrumadora presencia de comunicaciones en lengua japonesa, o en otras lenguas pero comunicaciones provenientes de hospitales japoneses ${ }^{4-6}$. Aizawa et al. ${ }^{5}$ en 1998 revisan la literatura japonesa recopilando 28 casos y el predominio continúa desde entonces.

La fusión de los tejidos vulvares parece estar en clara relación con déficit estrogénico que se produce en la mujer posmenopáusica ${ }^{2,7}$, la reducción o cese de la actividad sexual, así como factores en relación a procesos dermatológicos inflamatorios inespecíficos o específicos (liquen esclero atrófico, herpes genital), mala higiene local, etc. ${ }^{4,6}$
Normalmente la presencia de sintomatología urinaria en el interrogatorio parece orientar a otras posibilidades diagnósticas, pero la mera exploración da el diagnóstico. Normalmente una valoración somera del aparto urinario se impone.

La imposibilidad de recogida de orina impiden su estudio y el cultivo de la misma se deberán posponer hasta que el acceso al meato uretral esté expedito o la paciente reanude micciones fisiológicas. Una infección urinaria acompañante puede estar presente.

El tratamiento quirúrgico bajo actuación anestésica (anestesia espinal, analgo-sedación etc.) se impone, no presentando normalmente problemas, lográndose por mera disección roma la separación de los tejidos fusionados. La posibilidad de recidiva de la fusión debe ser motivo de máxima vigilancia, instaurando una higiene personal o asistida muy esmerada y la aplicación de pomadas con estrógenos.

\section{REFERENCIAS}

1. Fernández Larrañaga A, Comesaña Dávila E. Reflujo uretrovaginal consecutivo a la fusión de los labios menores. Actas Urol Esp. 1985;9(2):183-184.

2. Schober J, Dulabon L, Martin-Alguacil N, Kow LM, Pfaff D. Significance of topical estrogens to labial fusion and vaginal introital integrity. J Pediatr Adolesc Gynecol. 2006;19(5):337339.

3. Varenhorst E. Vulvar fusion. Unusual cause of urinary obstruction. Eur Urol. 1978;4(3):222-223.

4. Kato K, Kondo A, Takita T, Mitsuya H. Labial adhesions in a diabetic woman. Urol Int. 1986;41(6):455-456.

5. Aizawa T, Ishibashi K, Oyama H, Samejima T, Miki M. A case of labial adhesions in an elderly woman. Hinyokika Kilo. 1998;44(2):129-132.

6. Tsukigi M, Kubota Y, Onmura Y, Mitsuhashi Y, Tomita Y. A case of labial adhesions due to lichen sclerosus et atrophicus. Int Urol Nephrol. 2004;36(2):217-218.

7. Corroy JS, Girot V, Amicabile C, Guillemin P. Synechie des petites lévres cause de dysurie. Ann Urol. 1989;23(6):504-505.

Correspondencia autor: Dr. M.A. López Aramburu

Servicio de Urología

Hospital Santiago Apóstol

Ctra. de Orón, s/n - 09200 Miranda de Ebro (Burgos)

Tel.: 947349000

E-mail autor: malaramburu@yahoo.es

Información artículo: Nota clínica

Trabajo recibido: marzo 2007

Trabajo aceptado: mayo 2007 